\title{
Adsorción de la dureza del agua sobre lechos de rocas volcánicas de Ecuador
}

\author{
José G. Prato1, 2, Luisa C. González-Ramírez ${ }^{3}$, Maribel C. Pérez ${ }^{4}$ y Miguel E. Rodríguez ${ }^{5}$ \\ (1) Facultad de Ingeniería. Ingeniería Ambiental. Universidad Nacional de Chimborazo. Grupo de Estudios \\ Interdisciplinarios. Riobamba, Ecuador. (Correo-e: jose.prato@unach.edu.ec) \\ (2) Facultad de Ingeniería, Esc. de Ingeniería Química. Universidad de Los Andes. Mérida. Venezuela \\ (3) Facultad de Ciencias de la Salud. Laboratorio Clínico, Grupo de Investigación Análisis de Muestras Biológicas y \\ Forenses. Universidad Nacional de Chimborazo, Riobamba, Ecuador. (Correo-e: Icgonzalez@unach.edu.ec) \\ (4) Facultad de Ingeniería. Ingeniería Industrial. Universidad Nacional de Chimborazo. Riobamba, Ecuador. \\ (Correo-e: maribel.perez@unach.edu.ec) \\ (5) Facultad de Ciencias de la Educación, Humanas y Tecnologías, Universidad Nacional de Chimborazo, Campus "La \\ Dolorosa", Riobamba, Ecuador. (Correo-e: eduardo.rodriguez@unach.edu.ec)
}

* Autor a quien debe ser dirigida la correspondencia

Recibido Ago. 26, 2020; Aceptado Nov. 4, 2020; Versión final Dic. 4, 2020, Publicado Abr. 2021

\begin{abstract}
Resumen
La presente investigación muestra los resultados del proceso de adsorción de la dureza del agua empleando lechos fijos, con rocas de los volcanes Tungurahua y Reventador. El estudio se realizó sobre el material natural y sustratos tratados químicamente en medio alcalino para generar cagas superficiales negativas. Se determinó la composición química, el área superficial, la porosidad y el pH de los materiales ígneos. A partir de las pruebas de adsorción se comprobó que la capacidad de retención de la dureza depende del contenido de óxidos anfóteros de hierro, aluminio y titanio, siendo el lecho del volcán Tungurahua el que presenta mayor rendimiento $(65.5 \%)$ con respecto al Reventador (41.6\%). Se concluye que este sistema de adsorción representa una alternativa innovadora, de forma sencilla, económica y sustentable para el tratamiento de aguas duras y que puede ser aplicado a las comunidades de bajos recursos para mejorar la calidad del agua de uso doméstico y agropecuario.
\end{abstract}

Palabras clave: adsorción; dureza; agua; rocas volcánicas; lecho fijo; óxidos anfóteros

\section{Water hardness adsorption in volcanic rock beds from Ecuador}

\begin{abstract}
The primary objective of the present research study was to characterize the process of water hardness adsorption in rocks from the Tungurahua and Reventador volcanoes (Ecuador) by using fixed beds. The study was performed on natural material and chemically treated substrates in an alkaline medium to generate negative surface sites. The chemical composition, surface area, porosity, and $\mathrm{pH}$ of igneous materials were determined. From the adsorption tests, the results show that the hardness retention capacity depends on the total contents of amphoteric oxides of iron, aluminum, and titanium. The Tungurahua volcano bed showed a higher yield $(65.5 \%)$ than the Reventador bed $(41.6 \%)$. It is concluded that the adsorption system examined here is an innovative, simple, economic, and sustainable alternative for treating hard water that can be applied to improve water quality for domestic and agricultural use in low-income communities.
\end{abstract}

Keywords: adsorption; water hardness; volcanic rocks; packed-bed; amphoteric oxides 


\section{INTRODUCCIÓN}

Según la Organización Mundial de la Salud (WHO, 2010) la dureza del agua se puede definir como la medida de la capacidad del agua para reaccionar con el jabón y producir un depósito notable de precipitado (por ejemplo, metales o sales insolubles). La principal causa de la dureza del agua natural es la presencia de iones de Calcio $\left(\mathrm{Ca}^{2+}\right)$ y Magnesio $\left(\mathrm{Mg}^{2+}\right)$. Otros iones bivalentes que también participan en la dureza son hierro $\left(\mathrm{Fe}^{2+}\right)$, Manganeso $\left(\mathrm{Mn}^{2+}\right)$ y Estroncio $\left(\mathrm{Sr}^{2+}\right)$ principalmente, aunque no en cantidades significativas. La forma estándar de expresar la dureza del agua es en miligramos de carbonato de calcio equivalente por litro $(\mathrm{mg} / \mathrm{L}$ $\mathrm{CaCO}_{3}$ ) o de ppm de $\mathrm{CaCO}_{3}$, considerándose agua dura aquella que excede los $60 \mathrm{mg} / \mathrm{L} \mathrm{CaCO}$ de concentración. Estas aguas poseen algunas desventajas, afecta las redes de distribución urbanas (debido a la aparición de incrustaciones internas en las tuberías) y los sistemas de riego agrícolas. Además, la precipitación de calcio, altera la composición química del suelo, impidiendo la acción correcta de los agroquímicos en los cultivos (Sahin et al., 2012).

Los estudios epidemiológicos son controversiales, sin embargo, han demostrado el riesgo de enfermedad cardiovascular por el depósito del calcio en las paredes de los vasos sanguíneos, que unido a los lípidos producen el endurecimiento de las paredes vasculares (aterosclerosis) y la reducción de la luz de las arterias que dificulta el flujo sanguíneo (Sengupta, 2013). También se ha reportado el retraso en el crecimiento de los niños, debido a la interferencia del exceso de calcio con la absorción de minerales esenciales (Sengupta, 2013). Además, el contacto prolongado con agua dura, produce dermatitis atópica por deterioro de la barrera cutánea después de la exposición a los tensoactivos de los productos de lavado, combinado con altos niveles de calcio (Danby et al., 2018). Igualmente, se ha estudiado la relación entre la Enfermedad Renal Crónica de etiología incierta (KCDu, por sus siglas en ingles) y la dureza del agua en regiones rurales cercanas de Sri Lanka (Udeshani et al., 2020), y se ha encontrado una relación directa entre estas dos variables. Por tanto, reducir los niveles de $\mathrm{Ca}^{2+} \mathrm{y} \mathrm{Mg}^{2+}$ en el agua es un tema de importancia actual.

En la literatura se reportan varios métodos para reducir la dureza y/o filtrar el agua. En este sentido, Aregu et al. (2018) han estudiado rocas volcánicas tipo pómez y escoria de la región de Orimia en Etiopia para filtrar agua. Similarmente, se han empleado rocas volcánicas para la retención de metales como cromo y cadmio, encontrando que son materiales prominentes para remover estos metales del agua dura (Alemayehu et al., 2011; Rueda et al., 2010). También, existen investigaciones con zeolitas naturales y modificadas (Liu, 2020; Zayed et al., 2017; Fu y Wang, 2011; Alemayehu et al., 2011), carbón activado (Dobrosz-Gómez et al., 2018; Colpas et al., 2016; Rojas et al. 2012; Moreno y Giraldo, 2008) y suelos lateríticos ricos en óxidos metálicos, que han demostrado la utilidad de los mismos en el tratamiento de aguas contaminadas con cationes y aniones disueltos, variando su eficiencia en función de las características morfológicas de los lechos adsorbentes, el contenido y tipo de mineral presente en estos materiales (Márquez et al., 2020; Prato et al., 2019; Millán et al., 2009, 2019).

La Cordillera Real del Ecuador es una cadena volcánica de $225 \mathrm{~km}$ de largo, formada en general por volcanes andesíticos activos, potencialmente activos e inactivos (Hall et al., 2008), donde predominan rocas ígneas que se formaron por el enfriamiento de lava en la superficie terrestre, las cuales presentan diferentes características, dependiendo de la composición mineralógica y estructura geológica de cada zona. Los minerales presentes en los materiales litológicos pueden ser aprovechados como centros activos de adsorción e intercambio iónico para retener los iones calcio y magnesio que originan la dureza de las aguas naturales. Si bien, las rocas ígneas del Ecuador han sido estudiadas desde el punto de vista morfológico y edafológico (Mothes, 1991, Hall et al., 1999; 2008; Bablon et al., 2017), no hay trabajos específicos que las utilicen en la fabricación de lechos adsorbentes para la retención sales disueltas.

Ecuador, por su ubicación geográfica y ambientes geológicos presenta un complejo estructural heterogéneo formado por rocas metamórficas (INIGEMM, 2017), por lo tanto, las reservas de aguas naturales de estas zonas presentan diversos aniones y cationes, entre los que se destaca los iones de calcio y magnesio. En los acuíferos subterráneos las sustancias químicas disueltas dependen del depósito geológico del lugar por el que han fluido. En la Región Sierra (zona central del país) el agua generalmente es captada de fuentes naturales que tienen concentraciones de dureza variables entre 150 y $407.67 \mathrm{mg} / \mathrm{L} \mathrm{CaCO}$. Así, la población solo tiene acceso a aguas duras de concentración promedio de $336 \mathrm{mg} / \mathrm{L} \mathrm{CaCO} 3$ (Romero, 2009), niveles que superan el máximo permitido por la legislación nacional de $300 \mathrm{mg} / \mathrm{L} \mathrm{CaCO}{ }_{3}$ (NTE 1108, 2014), estando permanentemente expuestos a los riesgos asociados a la dureza del agua como podría ser al KCDu.

Por tanto, en este trabajo se pretende estudiar la aplicación de yacimientos de rocas de 2 volcanes de Ecuador (Reventador y Tungurahua), en la preparación de lechos adsorbentes, para la remoción de la dureza del agua. Esto permitirá evaluar su composición química y características físicas, así como determinar la capacidad de adsorción catiónica de estos materiales de forma natural y activada, para potenciar a futuro su uso como un sistema de tratamiento de bajo costo aplicable a las comunidades rurales. 


\section{MATERIALES Y MÉTODOS}

El estudio fue de enfoque cuantitativo, cohorte transversal, con un nivel de alcance correlacional y explicativo y un diseño cuasiexperimental. La metodología empleada en la investigación es presentada en tres subsecciones que comprenden: la descripción del área de muestreo, la preparación y caracterización de los lechos de adsorción y el procedimiento utilizado en las pruebas de retención de la dureza del agua.

\section{Descripción del área de muestreo}

Se han estudiado rocas volcánicas de 2 regiones diferentes de Ecuador, materiales ígneos que presentan una mineralogía diversa que va en función de su composición química y de las condiciones de temperatura y presión incidentes durante su formación: Volcán Reventador (Fig. 1) y Volcán Tungurahua (Fig.2).

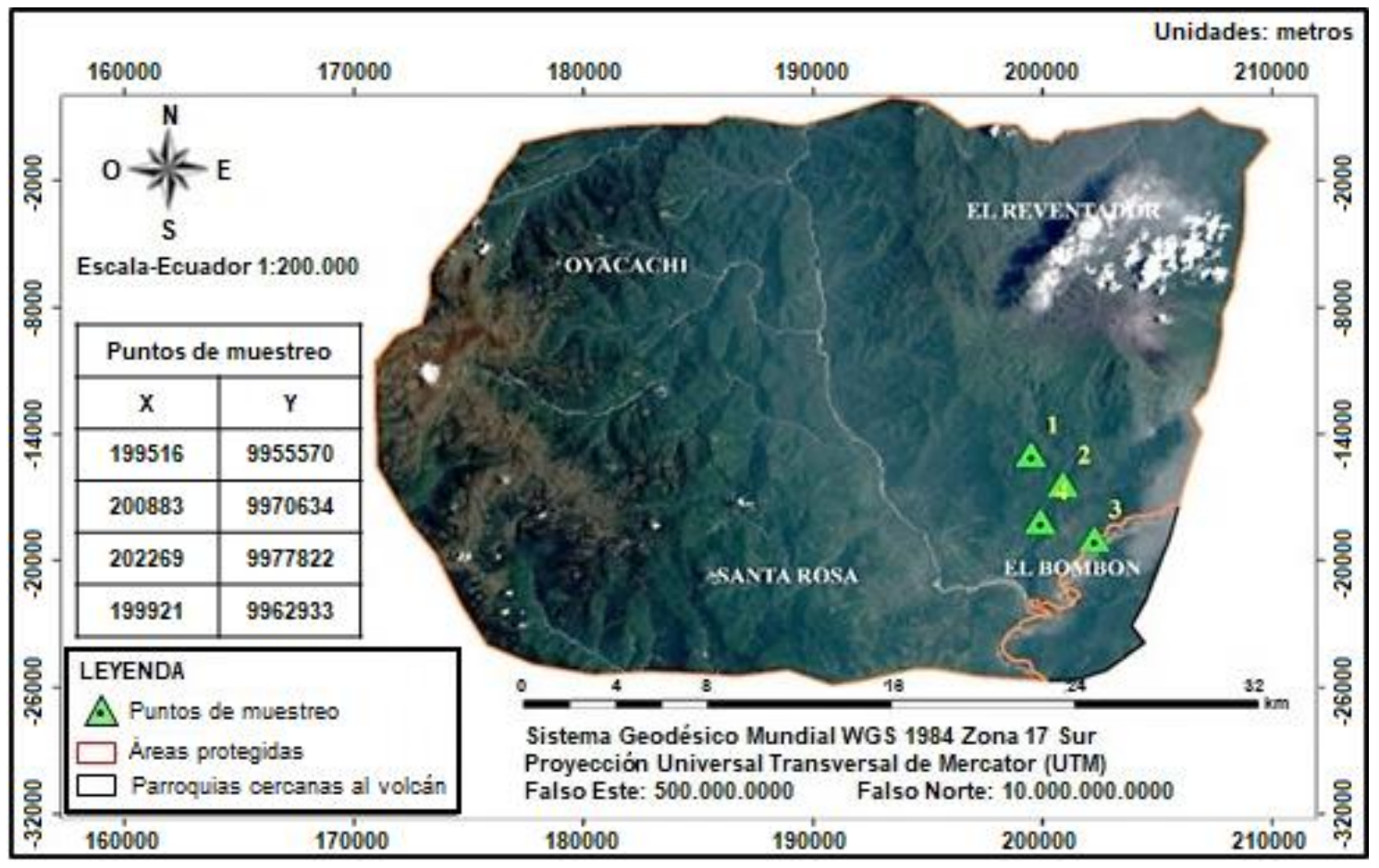

Fig 1: Área de muestreo del material volcánico del Reventador

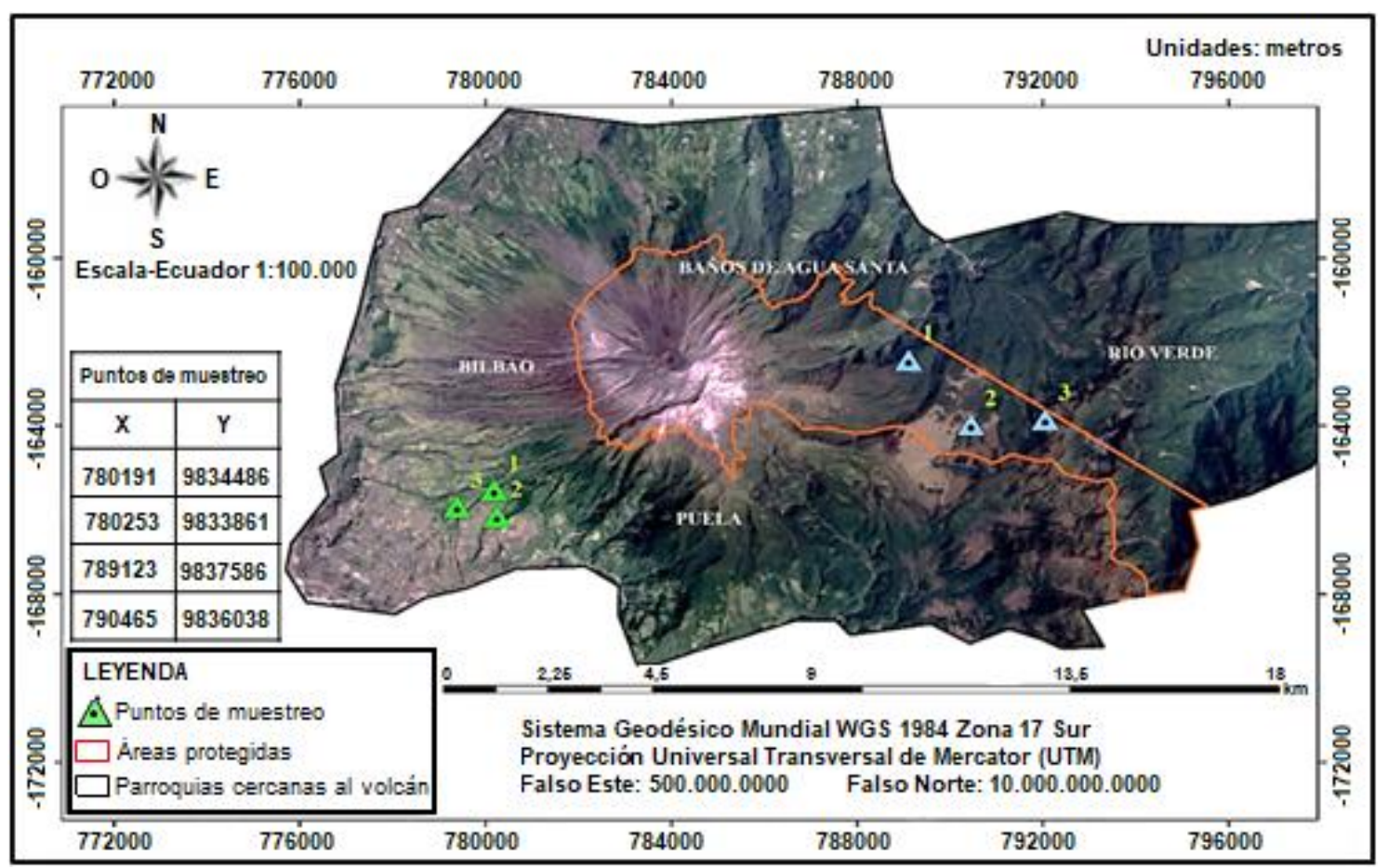

Fig. 2: Área de muestreo del material volcánico del Tungurahua 
El volcán Reventador, pertenece a la región oriental, tiene una altitud de 3562 m.s.n.m., formado principalmente por andesitas y andesitas basálticas que poseen minerales ferromagnésicos típicos del basalto de roca volcánica, de color negro verdoso, compuesto de feldespato y piroxeno (Mothes, 1991; Hall et al., 2008). Mientras que, el volcán Tungurahua localizado en la región central, con una altitud de 5023 m.s.n.m., se caracteriza por rocas de tipo andesitas y dacitas calco-alcalinas de medio potásico con carácter adakítico (Hall et al., 1999; Bablon et al., 2017). Ambos volcanes se categorizan como activos de reciente erupción. Se tomaron 4 muestras simples de rocas volcánicas en cada una de las regiones, referenciándose con un GPS cada punto por sector. Las muestras simples son mezcladas para obtener una muestra representativa y homogénea de cada volcán.

\section{Preparación y análisis fisicoquímico de los lechos de adsorción}

Los medios de adsorción se obtienen a partir de la trituración y tamizado de las rocas volcánicas hasta obtener partículas de $1 \mathrm{~cm}$ de tamaño promedio. Este material fraccionado se utilizó para realizar los análisis fisicoquímicos: i) Dimensiones promedio del lecho; ii) Área superficial BET y composición química elemental; y iii) Porosidad y $\Delta p H$.

Dimensiones promedio del lecho: para determinar el tamaño de grano promedio se escogió al azar una cantidad de 100 rocas de los lechos de adsorción de ambos volcanes en estudio, y se midió el largo, alto y ancho de cada roca.

Área superficial BET y composición química elemental: la superficie específica de los materiales se midió por el método de adsorción de $\mathrm{N}_{2}$ a la temperatura de $-196^{\circ} \mathrm{C}$, usando un equipo Micromeritics ASAP 2000. Las muestras (aproximadamente $200 \mathrm{mg}$ ) con un tamaño de partícula de $0.1-0.4 \mathrm{~mm}$, se trataron a $400^{\circ} \mathrm{C}$ y vacío durante 12 horas antes de las medidas de adsorción. La mineralogía de las muestras se determinó empleando un espectrómetro de fluorescencia de rayos-x (FRX) Phillips MiniPal $25 \mathrm{fm}$ analytic X-ray.

Porosidad y $\Delta \mathrm{pH}$ : la porosidad se determinó midiendo el volumen de agua absorbida por una muestra de lecho, pesándola antes y después de haber sido sumergida en agua hasta su saturación, la diferencia de peso entre la muestra seca y húmeda, es un indicador del volumen de agua contenida en el lecho saturado, siendo equivalente al volumen de los poros, por lo que se usa para determinar la porosidad del material (Millán et al., 2009), la cual se calcula según la ecuación (1). La carga predominante (positiva o negativa) de las rocas volcánicas se midió a través del $\Delta \mathrm{pH}$ del material triturado, calculando la diferencia entre el $p \mathrm{H}$ en agua destilada y el $p \mathrm{H}$ en $\mathrm{KCl}$ a una concentración de $1 \mathrm{~N}$ (Tan, 2011).

$$
\% \text { porosidad }=\frac{\text { Volumen agua absorbida }}{\text { Volumen de la muestra }} * 100
$$

\section{Pruebas de adsorción}

Se diseñaron columnas de adsorción empleando $1050 \mathrm{~g}$ de rocas de cada uno de los volcanes de manera independiente en tuberías cilíndricas de PVC de $57 \mathrm{~cm}$ de alto y $13 \mathrm{~cm}$ de diámetro. Para evitar taponamientos se usaron mallas metálicas con forma de colador en el fondo de cada columna. Las pruebas se realizaron empleando dos tipos de lechos: naturales, sin ninguna modificación solamente lavados con agua destilada para eliminar el material suelto (lechos sin activar) y activados, es decir, lechos sumergidos en una solución de $\mathrm{NaOH}$ al $0.001 \mathrm{~N}$ por 30 minutos y lavados con agua destilada antes de su uso. Los experimentos se realizaron empleando una columna de lecho fijo con un caudal $100 \mathrm{~mL} / \mathrm{min}$. Inicialmente, la adsorción se realizó con una solución patrón de $400 \mathrm{mg} / \mathrm{L} \mathrm{CaCO}_{3}$, y luego se determinó la retención de la dureza del agua natural extraída de pozos subterráneos de la Universidad Nacional de Chimborazo. Se analizó la cantidad de dureza total del agua tratada que sale de la columna cada $50 \mathrm{~mL}$, hasta que su concentración retomó el valor de la muestra de entrada, es decir, hasta alcanzar la saturación del lecho. La dureza de las aguas se midió empleando el Método 2340 C Titulométrico con EDTA (APHA, AWWA, \& WPCF, 2017).

\section{RESULTADOS Y DISCUSIÓN}

Se presentan los resultados de la caracterización de las rocas volcánicas, así como, los estudios de adsorción de la dureza total empleando los lechos sin activar y activados.

\section{Caracterización fisicoquímica de los lechos de adsorción}

En la Tabla 1 se reportan los resultados de caracterización de los lechos triturados. En ésta se puede observar que los granos de los lechos preparados presentan una uniformidad en sus dimensiones, teniendo una longitud promedio de $1.15 \mathrm{~cm}$, de alto $0.63 \mathrm{~cm}$ y un ancho medio de $0.61 \mathrm{~cm}$. También, se registra una porosidad similar en ambos materiales volcánicos. El lecho pétreo del Reventador presenta menor área 
superficial que el material del Tungurahua, ambos registros se consideran bajos, lo que puede afectar el proceso de retención de la dureza total del agua, al ser tratada por adsorción sobre estos materiales.

Las rocas del Tungurahua y Reventador presentan un $\Delta p H$ positivo, aunque la diferencia de los resultados entre los volcanes es mínima. Este parámetro puede ser un indicador del rendimiento o eficiencia que tienen los lechos en la capacidad de adsorción de la dureza total del agua como se reporta en la literatura (Xu et al., 2016; Colpas et al., 2016; Tan, 2011; Moreno y Giraldo, 2008). En los materiales oxídicos, el fenómeno de adsorción depende de la formación de cargas variables en función del $p \mathrm{H}$ en sus superficies, y con $\Delta p \mathrm{H}$ positivo es mayor el intercambio aniónico que catiónico.

Tabla 1: Caracterización de los lechos de rocas volcánicas

\begin{tabular}{|c|c|c|c|c|c|c|}
\hline \multirow{2}{*}{$\begin{array}{c}\text { Lecho } \\
\text { Triturado }\end{array}$} & $\begin{array}{c}\text { Área superficial } \\
B E T\left(\mathrm{~m}^{2} / \mathrm{g}\right)\end{array}$ & $\begin{array}{c}\text { Porosidad } \\
(\%)\end{array}$ & \multirow{2}{*}{$\Delta \mathrm{pH}$} & \multicolumn{3}{|c|}{ Dimensiones $(\mathrm{cm})$} \\
\cline { 5 - 7 } & & & Largo & Alto & Ancho \\
\hline Tungurahua & 0.3053 & 30.25 & 0.237 & $1.18 \pm 0.19$ & $0.67 \pm 0.12$ & $0.68 \pm 0.14$ \\
\hline Reventador & 0.2068 & 29.84 & 0.248 & $1.12 \pm 0.12$ & $0.59 \pm 0.17$ & $0.55 \pm 0.16$ \\
\hline
\end{tabular}

En cuanto a la mineralogía, se determinan pequeñas diferencias entre ambos materiales, en la Fig. 3, se observa que los componentes mayoritarios del lecho del Reventador son los óxidos de silicio (38.1\%), aluminio (13.4\%), calcio $(5.7 \%)$ y hierro $(5.3 \%)$, mientras que, las rocas del Tungurahua se caracterizan por tener principalmente óxidos de silicio (51.2\%), aluminio (17.4\%), calcio $(7.2 \%)$, hierro $(7.1 \%)$, magnesio $(3.5 \%)$ y potasio $(1.5 \%)$.
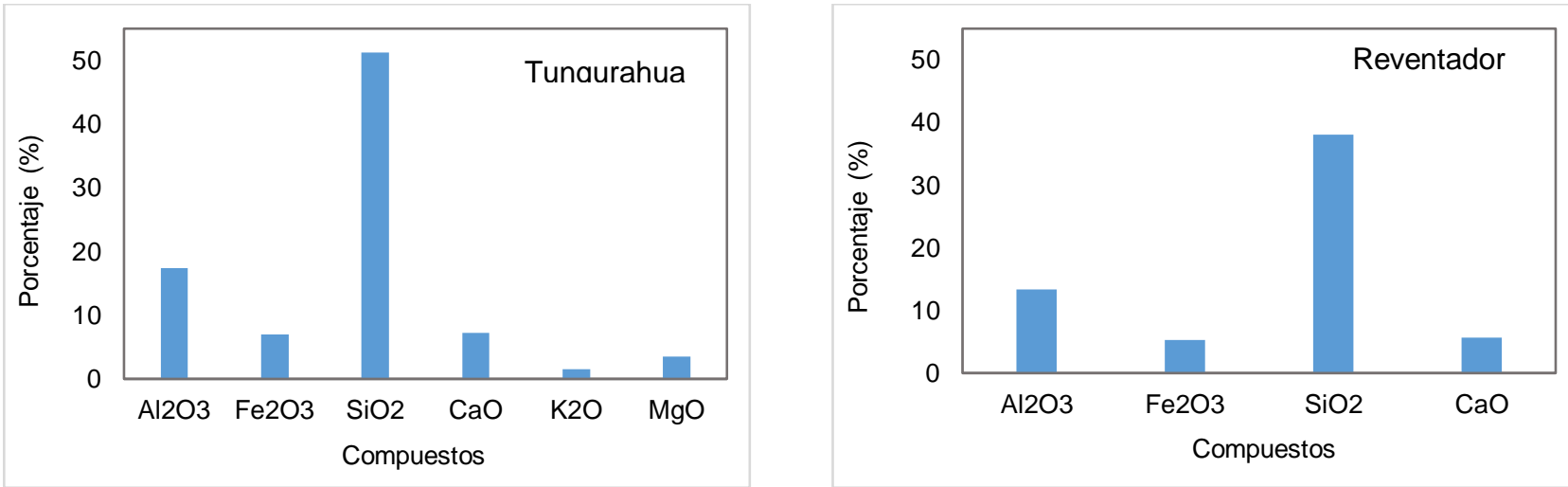

Fig 3: Componentes químicos mayoritarios de los lechos volcánicos

Además, en la Fig. 4 se pueden apreciar el resto de elementos químicos que conforman la estructura de los lechos (componentes minoritarios). El análisis muestra que ambos materiales están formados por una gran variedad de minerales. Aunque, los puntos GPS de muestreo de cada volcán (Fig. 1 y Fig. 2), permiten identificar similitudes en la formación geológica de los volcanes, donde predominan andesinas, plagidacitas y lavas que contienen gran cantidad de fenocristales de plagioclasas (Mothes, 1991; Hall, et al., 1999).
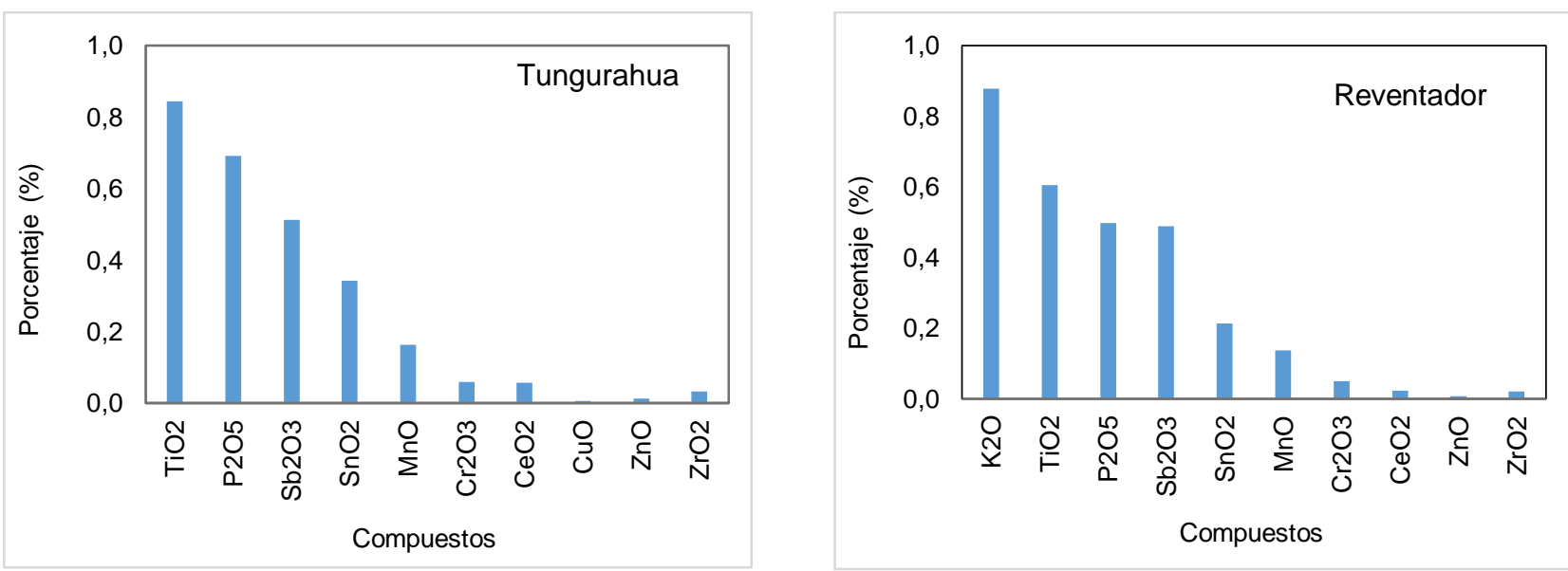

Fig. 4: Componentes químicos minoritarios de los lechos volcánicos 
La mineralogía obtenida en este estudio permite evidenciar que existen diferencias en la proporción de los metales comunes que se encuentran en ambos materiales, las rocas del volcán Tungurahua presentan un mayor contenido de $\mathrm{Fe}, \mathrm{Al}, \mathrm{Ti}, \mathrm{Mn}$, lo que puede favorecer la retención de la dureza del agua, debido a que los óxidos de estos metales contribuyen al fenómeno de adsorción por la formación de cargas variables dependientes del $\mathrm{pH}$ en las superficies de los materiales oxídicos (Márquez et al., 2020; Aragaw y Ayalew, 2019; Xu et al., 2016; Rueda et al., 2010). Además, estudios realizados con materiales lateríticos oxídicos indican que la retención de cationes (cobre, zinc, plomo, cromo, calcio), se ve favorecida por el contenido de elementos bivalentes y trivalentes que permiten cambiar la carga superficial, mejorando la adsorción de iones (Liu et al., 2020; Millán et al., 2019, Tan, 2011; Alemayehu et al., 2011).

\section{Estudios de adsorción}

A continuación, se reportan los resultados de las pruebas de retención de la dureza total del agua sintética usando lechos naturales y modificados químicamente, además del tratamiento del agua subterránea.

\section{Lechos sin activar}

En los ensayos con la solución patrón se observó que el lecho natural del volcán Tungurahua retiene inicialmente los iones de $\mathrm{Ca}^{2+}$ presentes en la solución, bajando la concentración de dureza del agua de 400 a $175 \mathrm{mg} / \mathrm{L} \mathrm{CaCO}_{3}$ (Fig. 5), luego disminuye su retención de forma escalonada a medida que el lecho se satura, logrando extraer la dureza de $2500 \mathrm{~mL}$, antes de perder su efectividad. Los resultados también indican, que una vez que el lecho se satura, se puede lavar con agua destilada, recuperando su actividad inicial y manteniendo el mismo rendimiento que un lecho natural nuevo. Por lo tanto, la retención de los iones $\mathrm{Ca}^{+2}$, sobre las rocas, es una adsorción de tipo física, débil, y de fácil desorción (Millán et al., 2009), permitiendo la rápida recuperación del sistema de adsorción, lo que favorece su posible aplicación.

El lecho de adsorción preparado con las rocas del Reventador (Fig. 5) presenta un comportamiento similar, al inicio del proceso se produce el máximo de retención de dureza, llegando a disminuirla hasta $310 \mathrm{mg} / \mathrm{L}$ $\mathrm{CaCO}_{3}$, para posteriormente saturarse al cabo de $2000 \mathrm{~mL}$ percolados. Igualmente, el material recupera su rendimiento inicial una vez que es regenerado por lavado con agua destilada.
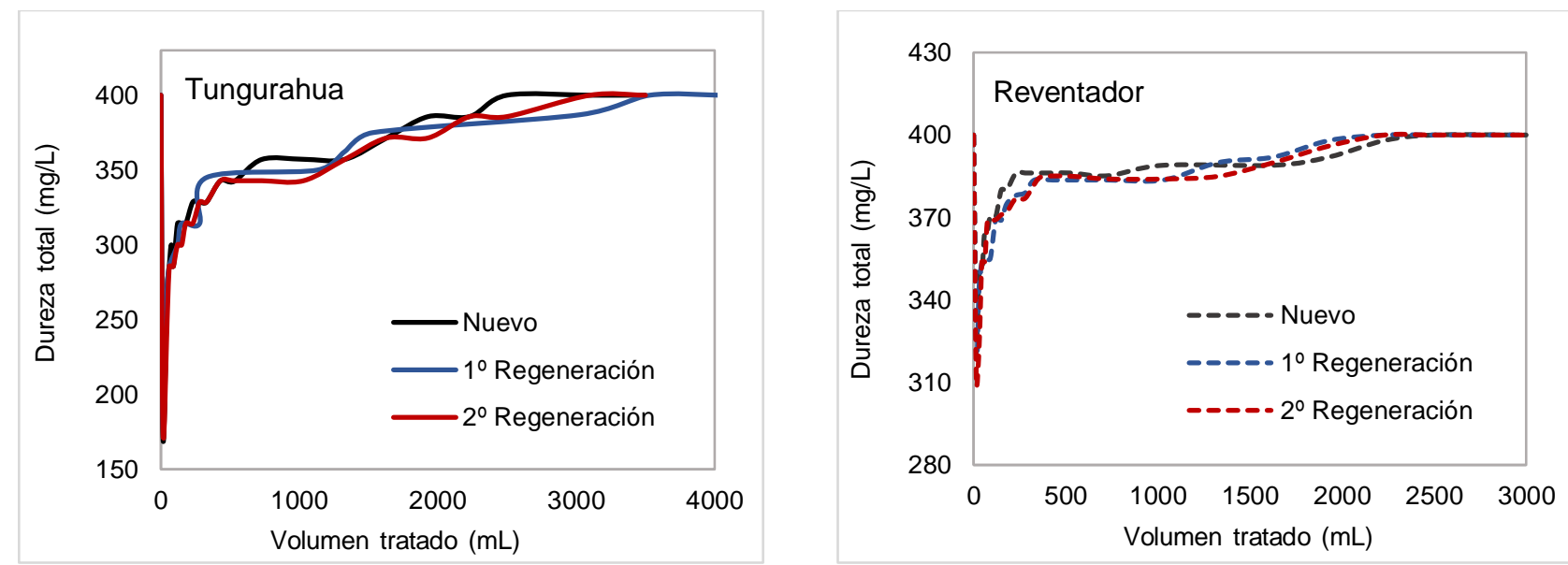

Fig 5: Reducción de la dureza total del agua empleando lechos sin activar

Los resultados indican que, el material ígneo del Tungurahua tiene una mayor capacidad de retención de la dureza del agua utilizada como solución patrón, logrando adsorber $3.05 \mathrm{mgCa}^{2+} / \mathrm{g}$ de lecho, lo que corresponde a un $24.9 \%$ de efectividad, mientras que, las rocas del Reventador adsorben $1.68 \mathrm{mgCa}{ }^{2+} / \mathrm{g}$ (14.3\%). La discrepancia obtenida en los rendimientos de ambos materiales se debe principalmente, a las diferencias de la composición química de las rocas, que permiten al Tungurahua una mejor adsorción al tener mayor cantidad de óxidos de aluminio, hierro y titanio, en relación al material del Reventador. Resultados similares han sido reportados en la disminución de iones disueltos en el agua al usarse suelos de carga variable, ricos en óxidos anfóteros, aumentando su capacidad de adsorción con el contenido de elementos metálicos (Márquez et al., 2020; Prato et al., 2019; Xu et al., 2016; Rueda et al., 2010). Otros factores que pueden estar influyendo en el resultado obtenido, son la carga superficial de los materiales ( $\Delta p \mathrm{H}$ positivo) y el área superficial BET, que afectan negativamente en la adsorción de la dureza, de allí que los rendimientos no superen el $25 \%$ (Tabla 1). La capacidad de adsorción podría ser mejorada si a las rocas se les aplica un tratamiento previo de deprotonación, creando una densidad de cargas superficial negativa que puede dar lugar a la adsorción catiónica (Xu et al., 2016; Tan, 2011; Millán et al., 2009). 


\section{Lechos Activados}

En la Fig.6 se muestra el resultado del proceso de adsorción de la dureza total del agua, después de activar los lechos volcánicos con una solución de $\mathrm{NaOH} 0.001 \mathrm{~N}$. El lecho construido con material del Tungurahua, logró retener $7.73 \mathrm{mgCa}^{2+} / \mathrm{g}$, aumentando el rendimiento de reducción de iones a $65.5 \%$. Simultáneamente, como consecuencia de la activación, se aumentó la capacidad del tratamiento de agua de 2500 a 8500 m, antes de que se sature el lecho. Para el material ígneo del Reventador, también, se observó incremento en la adsorción de la dureza del agua, llegando a $4.90 \mathrm{mgCa}^{2+} / \mathrm{g}(41.6 \%)$, alcanzando el nivel de saturación después de percolar $5000 \mathrm{~mL}$. Estos resultados indican que, la activación de los lechos mejora la reducción de la dureza del agua, debido a que la solución alcalina produce la deprotonación de los óxidos, generando cargas negativas que favorecen la adsorción catiónica (Márquez et al., 2020; Liu et al., 2020; Xu et al., 2016; Millán et al., 2009). Además, los resultados revelan que el sistema de adsorción es regenerable, sin perder efectividad, lo que indica la posibilidad de emplear soluciones alcalinas de fácil acceso y bajo costo para modificar las cargas superficiales de los lechos, incrementando su rendimiento y brindando una alternativa de aplicación de estos materiales en el tratamiento de aguas duras.
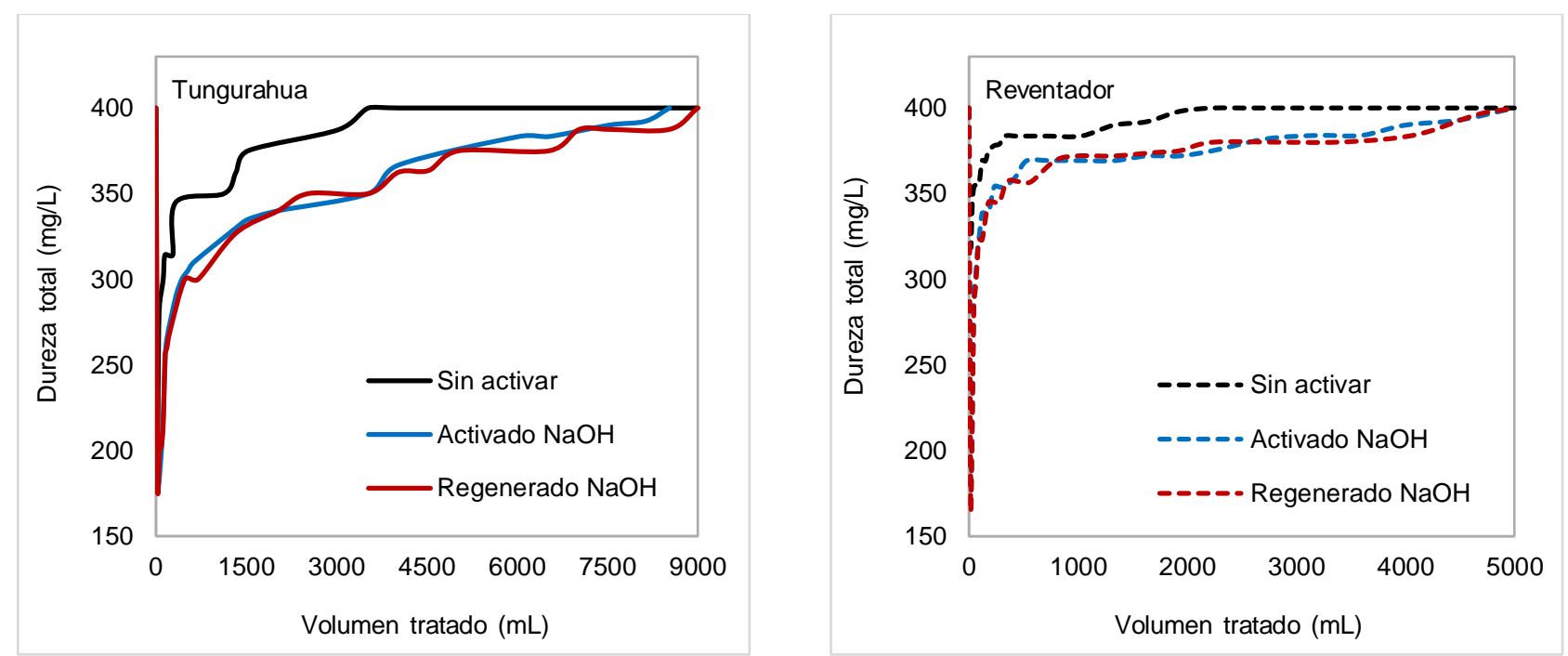

Fig. 6: Reducción de la dureza total del agua empleando lechos activados

\section{Ensayos con agua natural}

Finalmente, se evalúa la aplicación de los lechos de ambos volcanes en la reducción de la dureza del agua natural subterránea, lo que permite analizar la efectividad ante la presencia de otros iones disueltos en el agua, que pueden afectar el rendimiento del proceso de adsorción. En la Fig. 7, se evidencia que los dos tipos de rocas volcánicas activadas con $\mathrm{NaOH}$ disminuyen la dureza total del agua del pozo subterráneo desde los $380 \mathrm{mg} / \mathrm{L} \mathrm{CaCO}_{3}$, pasando por un máximo de reducción, $195 \mathrm{mg} / \mathrm{L} \mathrm{CaCO}_{3}$ en el caso del Reventador y 180 $\mathrm{mg} / \mathrm{L} \mathrm{CaCO}_{3}$ para el lecho del Tungurahua, también se puede observar que material volcánico del Tungurahua presenta una mayor efectividad, logrando tratar $3000 \mathrm{~mL}$ más de agua, antes de saturarse.

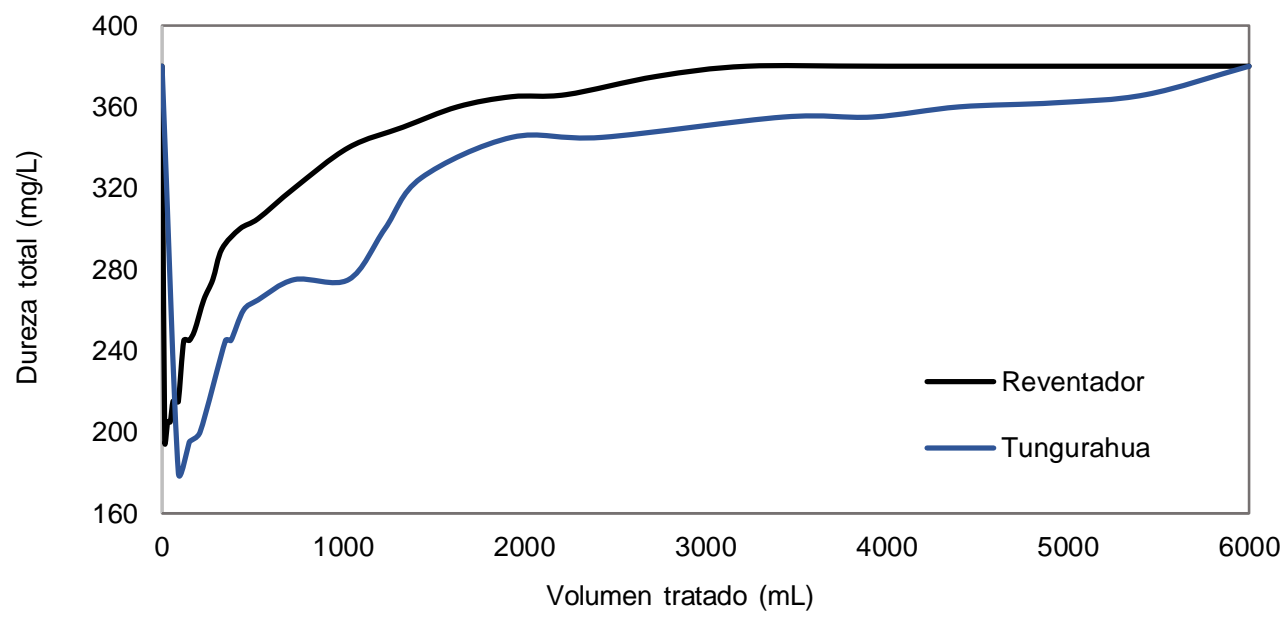

Fig. 8: Reducción de la dureza total de una muestra de agua natural 
Es importante destacar, que el rendimiento obtenido con el agua natural usando ambos materiales es levemente superior al obtenido con la solución patrón, lo que comprueba que la presencia de otras sales disueltas en el agua, no altera la capacidad de retención de la dureza, por competencia sobre los centros de adsorción de los lechos. Estos resultados demuestran que los lechos de roca volcánica (en especial del Tungurahua) son una alternativa de bajo costo y sencilla aplicación para la reducción de la dureza de las aguas naturales de la región.

\section{CONCLUSIONES}

De acuerdo al trabajo presentado y a los resultados obtenidos, se pueden plantear las siguientes conclusiones principales:

Los materiales probados de los volcanes Reventador y Tungurahua presentan una composición química variada, su capacidad de adsorción está relacionada principalmente, con la composición química del material, pudiéndose comprobar que, al tener mayor contenido de óxidos anfóteros de hierro, aluminio y titanio, se incrementa la retención de los iones que causan la dureza del agua.

Al deprotonizar los óxidos metálicos de las rocas volcánicas se incrementa la densidad de cargas negativas mejorando el rendimiento de adsorción, sin ser afectado por la presencia de otras sales disueltas en las aguas naturales, con la ventaja de poder ser regenerados para su reutilización.

Los resultados del trabajo demuestran que los lechos de adsorción preparados a partir de las rocas volcánicas presentan gran potencial para ser usados como sustratos adsorbentes de la dureza del agua, pudiendo ser una alternativa rentable y sustentable al tratamiento de aguas naturales, que puede ser aplicado a las comunidades de bajos recursos para mejorar la calidad del agua de uso doméstico y agropecuario.

\section{AGRADECIMIENTOS}

Los autores agradecen a la Dirección de Investigación y el Vicerrectorado de Investigación, Vinculación y Posgrado de la UNACH, por el apoyo a este trabajo con los proyectos: "Lechos de materiales oxídicos como medios de adsorción y filtración para el tratamiento de la contaminación de aguas Naturales" y "Lechos de rocas volcánicas para reducir la dureza en aguas naturales". Se reconoce la colaboración del Instituto de Tecnología Química, CSIC-UPV (España), por la determinación de las propiedades superficiales y químicas de los adsorbentes.

\section{REFERENCIAS}

Alemayehu, E., Thiele-Bruhn, S. y Lennartz, B.; Adsorption Behaviour of $\mathrm{Cr}(\mathrm{VI})$ onto Macro and Micro-vesicular Volcanic Rocks from Water, https://doi.org/10.1016/j.seppur.2011.01.020, Sep. Purif. Technol., 78, 55-61 (2011)

APHA, AWWA, \& WPCF.; Standard Methods for the Examination of Water and Wastewater, $23^{\text {rd }}$ Ed, American Public Health Association-American Water Works Association-Water Pollution Control Federation Ed., Washington, DC., USA (2017)

Aragaw, T.A. y Ayalew, A.A.; Removal of Water Hardness using Zeolite Synthesized from Ethiopian Kaolin by Hydrothermal Method, https://doi.org/10.2166/wpt.2018.116, Water Pract. Technol., 14(1), 145-159 (2019)

Aregu, M.B., Asfaw, S.L. y Khan, M.M.; Identification of Two Low-Cost and Locally Available Filter Media (Pumice and Scoria) for Removal of Hazardous Pollutants from Tannery Wastewater. https://doi.org/10.1186/s40068-018-0112-2, Environ. Syst. Res., 7, 10 (2018)

Bablon, M., Quidelleur, X. y otros 5 autores; Eruptive Chronology of Tungurahua Volcano (Ecuador) Revisited Based on New K-Ar Ages and Geomorphological Reconstructions, https://doi.org/10.1016/j.jvolgeores.2018.05.007, J. Volcanol. Geoterma. Res., 357(15), 378-398 (2018)

Colpas, F., Tarón, A.A., y Fon, W.; Adsorción de Mercurio Utilizando Carbones Activados Modificados con Peróxido de Hidrógeno y Calentamiento, http://dx.doi.org/10.4067/S0718-07642016000500009, Inf. tecnol., 27(5), 69-76 (2016)

Danby, S.G., Brown, K. y otros 5 autores; The Effect of Water Hardness on Surfactant Deposition after Washing and Subsequent Skin Irritation in Atopic Dermatitis Patients and Healthy Control Subjects, https://doi.org/10.1016/j.jid.2017.08.037, J. Invest. Dermatol., 138(1), 68-77 (2018)

Dobrosz-Gómez, I., Gómez, M.A., y Santa, C.; Optimización del Proceso de Adsorción de Cr(VI) sobre Carbón Activado de Origen Bituminoso, http://dx.doi.org/10.4067/S0718-07642018000600043, Inf. tecnol., 29(6), 43-56 (2018)

Fu F. y Wang Q.; Removal of Heavy Metal lons from Wastewaters: a Review, https://doi.org/10.1016/j.jenvman.2010.11.011, J. Environ. Manag., 92(3) 407-418 (2011)

Hall, M.L., Samaniego, P. y otros 2 autores; Ecuadorian Andes Volcanism: A Review of Late Pliocene to Present Activity, https://doi.org/10.1016/j.jvolgeores.2008.06.012, J. Volcanol. Geoterma. Res., 176(1), 1-6 (2008) 
Hall, M.L., Robin, C. y otros 3 autores; Tungurahua Volcano, Ecuador: Structure, Eruptive History and Hazards, https://doi.org/10.1016/S0377-0273(99)00047-5, J. Volcanol. Geoterma. Res., 91(1), 1-21 (1999)

Instituto Nacional de Investigación Geológico Minero Metalúrgico - INIGEMM. Atlas Geológico Minero de Ecuador. 4-22, INIGEMM, Quito, Ecuador (2017)

Liu, W., Singh, R.P. y otros 2 autores; Evaluation of Groundwater Hardness Removal using Activated Clinoptilolite, https://doi.org/10.1007/s11356-019-06193-9, Environ. Sci. Pollut. Res., 27(15), 17541-17549 (2020)

Márquez, A., Millán, F. y otros dos autores; Adsorción de lones $\mathrm{Cr}(\mathrm{VI})$ sobre Lechos Adsorbentes Calcinados con Superficie de Carga Variable Químicamente Modificada, https://doi.org/10.22209/rt.v43n2a03, Rev. Tec. Ing. Univ. Zulia, 43(2), 72-81, (2020)

Millán, F., Prato, J.G., y otros 2 autores; Estudio de la Retención de lones de Calcio por Materiales Térmicamente Modificados Provenientes de Suelos de la Región de San Juan de Lagunillas, Estado Mérida, Venezuela, http://ve.scielo.org/scielo.php?pid=S0254-07702009000100007\&script=sci_arttext, ISSN 0254-0770, Rev. Téc. Ing. Univ. Zulia, ISSN: 0254-0770, 32(1), 48-54 (2009)

Millán, F., Prato, J.G. y otros 3 autores; $\mathrm{Cu}^{+2}$ Chemisorption on Calcined Substrates Made with an Oxidic Refractory Variable Charges Lithological Material, https://produccioncientificaluz.org/index.php/tecnica/issue/view/2723/showToc, ISSN: 0254-0770, Rev. Téc. Ing. Univ. Zulia, 42(1), 10-18 (2019)

Moreno, J.C. y Giraldo, L.; Propiedades Termodinámicas del Proceso de Adsorción de $\mathrm{Pb}^{2+}$ sobre Carbón Activado a Diferentes pH, http://dx.doi.org/10.4067/S0718-07642008000500008, Inf. tecnol., 19(5), 61-72, (2008)

Mothes, P. El Paisaje Volcánico de la Sierra Ecuatoriana: Geomorfología, Fenómenos Volcánicos y Recursos Asociados, Corporación Editora Nacional, Quito, Ecuador (1991)

NTE INEN 1108. Instituto Ecuatoriano de Normalización, Norma Técnica Ecuatoriana, Agua Potable: Requisitos, $5^{a}$ Revisión, 1-10, Quito, Ecuador (2014)

Prato J.G., Ruiz, L.B., y otros 2 autores; Adsorción de lones Fosfatos de Aguas Naturales a partir de Lechos Calcinados de Suelos Lateríticos, In Investigación y Academia: la Visión desde la Universidad Ecuatoriana, by J.C. Arboleda (Ed), pp 133-151, REDIPE Red Iberoamericana de Pedagogía, Estados Unidos (2019)

Rojas, H.A., Guerrero, D.C., Vásquez, O.Y., y Valencia, J.S.; Aplicación del Modelo de Bohart y Adams en la Remoción de Mercurio de Drenajes de Minería por Adsorción con Carbón Activado, http://dx.doi.org/10.4067/S071807642012000300004, Inf. tecnol., 23(3), 21-32 (2012)

Romero, C.; "Optimización de la Red N² del Sistema de Agua Potable de la Ciudad de Riobamba”, Tesis de Grado, Esc. Ing. Química, Escuela Superior Politécnica de Chimborazo, Ecuador (2009)

Rueda, M.L., Volzone, C., Y Martínez., S.Y.; Adsorción de Cadmio en Solución Utilizando como Adsorbente Material Tobáceo Modificado, http://dx.doi.org/10.4067/S0718-07642010000400010, Inf. tecnol., 21(4) 75-78 (2010)

Sahin, U., Tunc, T. y Eroglu, S.; Evaluation of $\mathrm{CaCO}_{3}$ Clogging in Emitters with Magnetized Saline Water, https://doi.org/10/5004/dwt.2012.2845, Desalination Water Treat., 40, 168-173 (2012)

Sengupta, P.; Potential Health Impacts of Hard Water, https://www.ncbi.nlm.nih.gov/pmc/articles/PMC3775162/, Int. J. Prev. Med., 4(8), 866-875 (2013)

Tan, K.H.; Principles of Soil Chemistry, 4ª Ed., 133-241, CRC Press, New York, USA (2011)

Udeshani, W.A.C., Dissanayake, H.M., y otros 2 autores; Assessment of Groundwater Quality Using Water Quality Index (WQI): A Case Study of a Hard Rock Terrain in Sri Lanka, https://doi.org/10.1016/j.gsd.2020.10042, Groundw. Sustain. Dev., 11, 100421 (2020)

World Health Organisation - WHO; Hardness in Drinking-Water: Background Document for Development of WHO Guidelines for Drinking-Water Quality, WHO Press, Geneva, Switzerland (2010)

Xu, R.K, Qafoku N.P., y otros 3 autores; Adsorption Properties of Subtropical and Tropical Variable Soils: Implication from Climate Change and Biochard Amendment, https://doi.org/10.1016/bs.agron.2015.09.001, Adv. Agron., 135, 1-58 (2016).

Zayed A.M, Selim, A.Q., y otros 4 autores; Adsorption Characteristics of Na-A Zeolites Synthesized from Egyptian Kaolinite for Manganese in Aqueous Solutions: Response Surface Modeling and Optimization, https://doi.org/10.1016/j.clay.2017.01.027, Appl. Clay Sci., 140, 17-24 (2017) 
